\title{
Journal of Global Optimization Best Paper Award for 2015
}

\author{
Sergiy Butenko ${ }^{1}$
}

Published online: 21 October 2016

C Springer Science+Business Media New York 2016

I am very pleased to announce that the winners of the fifth annual Journal of Global Optimization Best Paper Award are Andrei Patrascu and Ion Necoara for their paper titled Efficient random coordinate descent algorithms for large-scale structured nonconvex optimization [5].

\begin{tabular}{l}
\hline Journal of Global Optimization Best Paper Award for 2015 \\
Authors: Andrei Patrascu and Ion Necoara \\
convex optimization \\
In this paper we analyze several new methods for solving nonconvex optimization prob- \\
lems with the objective function consisting of a sum of two terms: one is nonconvex \\
and smooth, and another is convex but simple and its structure is known. Further, we \\
consider both cases: unconstrained and linearly constrained nonconvex problems. For \\
optimization problems of the above structure, we propose random coordinate descent \\
algorithms and analyze their convergence properties. For the general case, when the \\
objective function is nonconvex and composite we prove asymptotic convergence for \\
the sequences generated by our algorithms to stationary points and sublinear rate of \\
convergence in expectation for some optimality measure. Additionally, if the objective \\
function satisfies an error bound condition we derive a local linear rate of convergence \\
for the expected values of the objective function. We also present extensive numeri- \\
cal experiments for evaluating the performance of our algorithms in comparison with \\
state-of-the-art methods.
\end{tabular}

The award consists of $\$ 1000$ and a commemorative plaque, and the award selection committee is comprised of several members of the editorial board. This year, Immanuel Bomze, Matthias Ehrgott, Oleg Prokopyev, Nikolaos Sahinidis, and Fabio Schoen served on the committee. We received record 27 nominations, 7 of which were selected as finalists. Based on the committee's votes, the following papers are recognized as "Top-5 Papers Published in Journal of Global Optimization in 2015":

\footnotetext{
Sergiy Butenko

butenko@tamu.edu

1 Texas A\&M University, College Station, TX, USA
} 
- Kerstin Dächert and Kathrin Klamroth, A linear bound on the number of scalarizations needed to solve discrete tricriteria optimization problems [2].

- Coralia Cartis, Jaroslav M. Fowkes, and Nicholas I. M. Gould, Branching and bounding improvements for global optimization algorithms with Lipschitz continuity properties [1].

- Peter J. C. Dickinson and Janez Povh, On an extension of Póya's Positivstellensatz [3].

- Ralph Baker Kearfott, Some observations on exclusion regions in branch and bound algorithms [4].

Congratulations to the award recipients and the authors of recognized papers on their excellent work! I would also like to thank Springer for sponsoring this award and the members of the award selection committee for their dedicated service. Please email your nominations for the 2016 Best Paper Award to butenko@ tamu.edu. All papers published in the journal in 2016 (volumes 64-66) are eligible.

\section{References}

1. Cartis, C., Fowkes, J.M., Gould, N.I.M.: Branching and bounding improvements for global optimization algorithms with Lipschitz continuity properties. J. Glob. Optim. 61, 429-457 (2015)

2. Dächert, K., Klamroth, K.: A linear bound on the number of scalarizations needed to solve discrete tricriteria optimization problems. J. Glob. Optim. 61, 643-676 (2015)

3. Dickinson, P.J.C., Povh, J.: On an extension of Pólya’s Positivstellensatz. J. Glob. Optim. 61, 615-625 (2015)

4. Kearfott, R.B.: Some observations on exclusion regions in branch and bound algorithms. J. Glob. Optim. 62, 229-241 (2015)

5. Patrascu, A., Necoara, I.: Efficient random coordinate descent algorithms for large-scale structured nonconvex optimization. J. Glob. Optim. 61, 19-46 (2015) 